\title{
Observation and control of collective spin-wave mode hybridization in chevron arrays and in square, staircase, and brickwork artificial spin ices
}

\author{
T. Dion $\odot,{ }^{1,2, *}$ J. C. Gartside $\odot,{ }^{3}$ A. Vanstone $\odot,{ }^{3}$ K. D. Stenning $\odot,{ }^{3}$ D. M. Arroo $\odot,{ }^{4,5}$ \\ H. Kurebayashi ${ }^{\circ},^{2}$ and W. R. Branford $\oplus^{3,4}$ \\ ${ }^{1}$ Solid State Physics Lab., Kyushu University, 744 Motooka, Nishi-ku, Fukuoka, 819-0395, Japan \\ ${ }^{2}$ London Centre for Nanotechnology, University College London, London WC1H OAH, United Kingdom \\ ${ }^{3}$ Blackett Laboratory, Imperial College London, London SW7 2AZ, United Kingdom \\ ${ }^{4}$ London Centre for Nanotechnology, Imperial College London, London SW7 2AZ, United Kingdom \\ ${ }^{5}$ Department of Materials, Imperial College London, London SW7 2AZ, United Kingdom
}

(Received 10 December 2021; accepted 4 January 2022; published 11 February 2022)

\begin{abstract}
Dipolar magnon-magnon coupling has long been predicted in nanopatterned artificial spin systems. However, observation of such phenomena and related collective spin-wave signatures have until recently proved elusive or been limited to low-power edge modes which are difficult to measure experimentally. Here we describe the requisite conditions for dipolar mode-hybridization, how it may be controlled, why it was not observed earlier, and how strong coupling may occur between nanomagnet bulk modes. We experimentally investigate four nanopatterned artificial spin system geometries: chevron arrays, square, staircase, and brickwork artificial spin ices. We observe significant dynamic dipolar-coupling in all systems with relative coupling strengths and avoided-crossing gaps supported by micromagnetic-simulation results. We demonstrate reconfigurable modehybridization regimes in each system via microstate control, and in doing so elucidate the underlying dynamics governing dynamic dipolar-coupling with implications across reconfigurable magnonics. We demonstrate that confinement of the bulk modes via edge effects plays a critical role in dipolar hybridized modes, and treating each nanoisland as a coherently precessing macro-spin or a standing spin-wave is insufficient to capture experimentally observed coupling phenomena. Finally, we present a parameter-space search detailing how coupling strength may be tuned via nanofabrication dimensions and material properties.
\end{abstract}

DOI: 10.1103/PhysRevResearch.4.013107

\section{INTRODUCTION}

Artificial spin ices (ASIs) are arrays of magnetically frustrated nanoislands with vast low-energy state degeneracy [1-4]. Study of ASI and related systems has expanded beyond modeling thermodynamic systems to leveraging them as host platforms for diverse applications including reconfigurable magnonics [1,3,5-10], neuromorphic [11,12] and reservoir computing [13-15]. Bypassing the need for parity of interactions, differential fabrication [16-19] offers enhanced tunability of the dynamic magnon response and increased microstate access flexibility. Reconfigurable magnonic crystals (RMCs) [6,20-31] are highly attractive due to hosting many distinct spin-wave spectra, with promising information-processing applications [32,33]. Magnonic crystals can express spin-wave band gaps, band-pass filtering, and waveguide bending [24,27]. Diverse functionality within the same RMC allows a plethora of different computational tasks and offers a potential solution to high power consump-

\footnotetext{
*troy.dion@phys.kyushu-u.ac.jp

Published by the American Physical Society under the terms of the Creative Commons Attribution 4.0 International license. Further distribution of this work must maintain attribution to the author(s) and the published article's title, journal citation, and DOI.
}

tion and waste heat [34] associated with traditional CMOS electronics. An attractive RMC avenue is engineering dipolar magnon-magnon coupling between nanomagnets. Typically, coupled magnetization dynamics is achieved via short-range exchange interaction [35,36], placing tight constraints on experimental system architecture. The dipolar-interaction responsible for coupling in nanopatterned RMCs offers relative freedom and reconfigurability of mode-hybridization phenomena [15]. There are many demonstrations of RMCs using 1D arrays $[21,23,25,26,26-30]$ which, while impressive, suffer from a limited number of states versus $2 \mathrm{D}$ arrays. ASIs are attractive to magnonic computing since they can be leveraged more flexibly and exhibit richer spin-wave spectra [5,15,16,31]. Rapid readout techniques for microstates have been developed for ASI, making it a promising RMC candidate [18,37].

Previously, dipole-dipole coupling and collective spinwave behavior in ASIs proved elusive and avoided crossings had not been observed. Interactions were considered too weak to resolve in ASI bulk modes (BMs) [38] or were limited to low-power edge-modes (EMs) [39,40], which are challenging to detect experimentally due to smaller magnetic volume and imperfect nanopatterned edges, although that can be improved using ion-beam milling [41]. Simulation of coupled nanomagnets in ASIs where interisland coupling is mediated by spin-wave channels in an exchange-biased underlayer has been demonstrated [42]. Here we show interisland dipolar 
coupling is sufficient for opening spin-wave band gaps using micromagnetic simulation (MuMax3 [43]) and experimental ferromagnetic resonance (FMR).

We previously investigated width-modified bicomponent square ASI, alternating rows of thin and wide nanoislands along each sublattice, termed staircase ASI. This provides access to type-3 states consisting of three-in, one-out vertex configuration whose spin-wave signature had yet been measured. Applying field $45^{\circ}$ to sublattice axes, we observed an avoided crossing due to antiparallel magnetization. This geometry with perpendicular state preparation and measurement field directions is atypical, and its use and efficacy in exploring mode-hybridization is further investigated here. We show collective spin-wave modes are not limited to geometrically-modified or 1D arrays and present a detailed study elucidating contributing factors to hybridized spin-wave modes in strongly-interacting nanomagnetic arrays. We investigate diagonal and chevron two nanoisland arrays, square, staircase, and brickwork ASI. A systematic parameter search is performed, including nanoisland-dimensions, vertex gap, array geometry and saturation magnetization for square ASI. The results shed light on dipolar magnon-magnon coupling and form a set of design rules for tailoring and controlling dipolar hybridization phenomena in artificial spin-system metamaterials [9]. Sample fabrication, experimental, fitting, and simulation methods are all found in the Supplemental Material [44].

\section{RESULTS AND DISCUSSION}

\section{A. Acoustic and optical spin waves}

Effective interisland coupling requires significant dynamic stray-field. Figure 1(a) illustrates different nanoisland spinwave modes; standing spin-wave modes (SSW) which exhibit insignificant stray-field, EMs predicted to exhibit modehybridization due to stray-field and BM which exhibit an uncharacteristic combination of EMs and SSWs with strayfield emanating from both short and long edges of the nanoisland allowing opportunity for effective inter-island coupling.

When moments are aligned parallel $(\uparrow \uparrow)$, no mode splitting occurs and both moments precess in phase [Fig. 1(b)]. When aligned antiparallel $(\uparrow \downarrow)$, mode-hybridization occurs and when the energies of BMs in separate nanoislands are brought close together an avoided-crossing is observed. We know from studies on synthetic antiferromagnets $[35,36,45,46]$ and bistable 1D nanoisland arrays $[21,23,25]$ that hybridizedmodes are distinguished by in-plane dynamic magnetization moving in-phase or out-of-phase termed acoustic and optical respectively as illustrated in Figs. 1(c) and 1(d). The out-of-plane dynamic magnetization has the opposite phase relationships, ie. acoustic moves out-of-phase and the optical moves in-phase due to opposite precession chirality. The frequency gap, $\delta$, is caused by Brillouin zone folding [21,25], typically occurring near remanence but can be shifted in field by width modification (staircase) or symmetry breaking (brickwork).

No strong interisland coupling exists if external fields are applied along sublattice directions [38]. Figure 1(e) illus- (a)

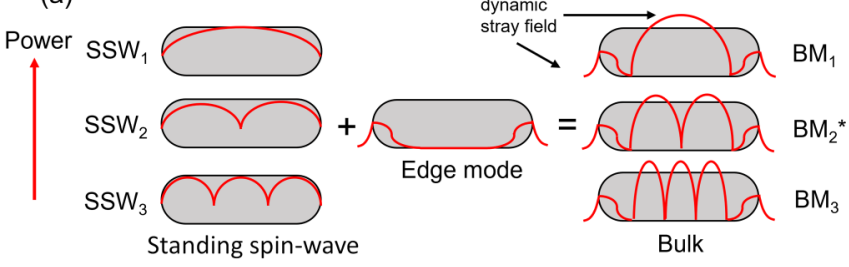

(b) In phase

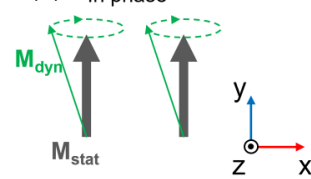

(c) In-phase (x-comp)

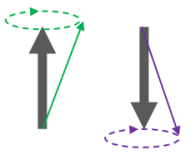

(acoustic)
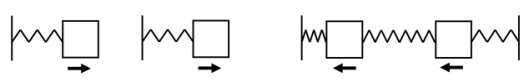

(e)

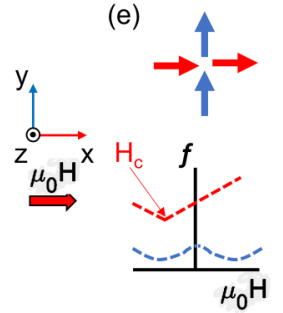

(f)

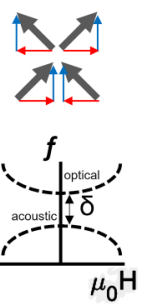

(d) Out-of-phase (x-comp)

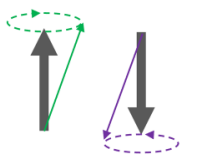

(optical)

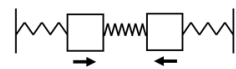

(g)
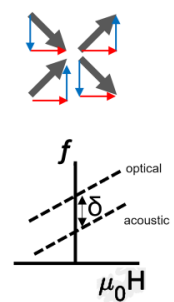

FIG. 1. (a) Power distributions of single nanoisland modes; standing spin-wave (SSW) modes, edge modes (EMs), and bulk modes (BMs). BMs observed experimentally and simulations are a combination of EMs and SSWs. Stray fields can emanate from long edges and short edges. *Not excited in uniform field. (b) Two parallel-magnetized nanoislands precess coherently, analogous to two uncoupled masses (shown underneath). (c) Acoustic mode where oppositely magnetized nanoislands' $x$ components of dynamic magnetization precess in phase, analogous to two spring-coupled masses moving coherently. (d) Optical mode where oppositely magnetized nanoislands' $x$ components move out of phase, analogous to two spring-coupled masses moving in opposite directions. (e) Type-2 ASI microstate with field applied parallel to sublattice. (f) Type-2 ASI magnetized perpendicular to the applied field direction. Hybridization occurs only near avoided crossing. (g) Type-2 ASI microstate with parallel preparation and measurement field.

trates the coercive field of the parallel-to-field nanoislands (red) are much lower than the perpendicular-to-field nanoislands (blue) and therefore the avoided crossings cannot be observed in principle. Figures 1(f) and $1(\mathrm{~g})$ show applying the field diagonally brings modes close together and each nanoisland magnetization can be broken down into two $x, y$ components. For hybridization between neighboring singlenanoisland modes to occur, a pair of nanoislands must have $\uparrow \downarrow$ configuration; in Fig. $1(\mathrm{~g}), x$ component $\rightleftharpoons$ (red) or Fig. 1(f), the $y$ component $\uparrow \downarrow$ (blue). It follows that avoided crossings should be observable in a field-saturated type-2 state if measurement and preparation fields are perpendicular. Typically, arrays are saturated along a given axis, then spectra measured while sweeping the field along the same axis, as illustrated in Fig. 1(e) [19,38,47,48], partially explaining why avoided crossings had not been observed before. The 


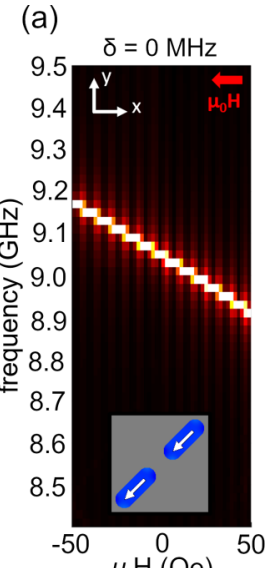

(b)
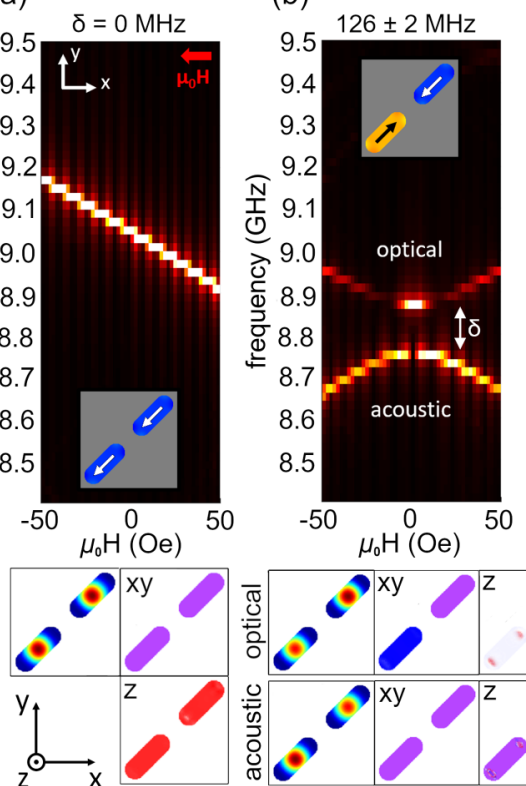

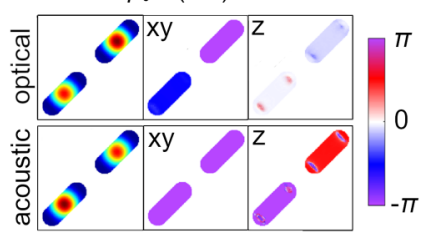

(c)

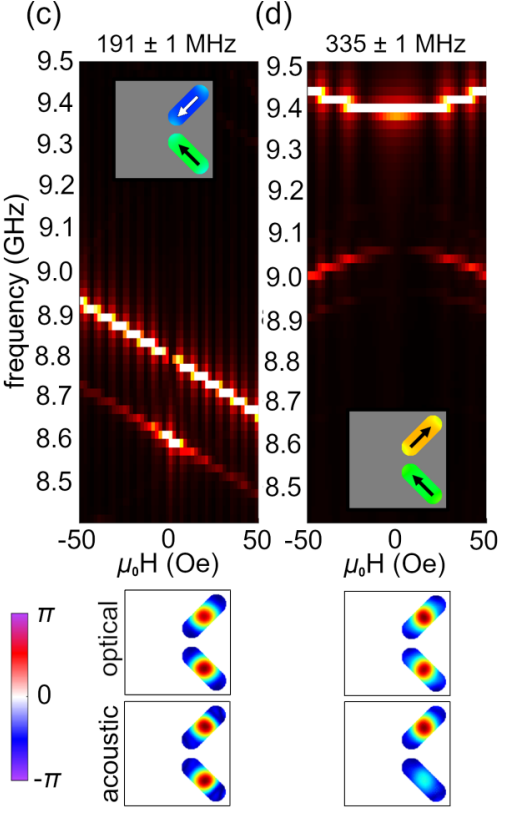

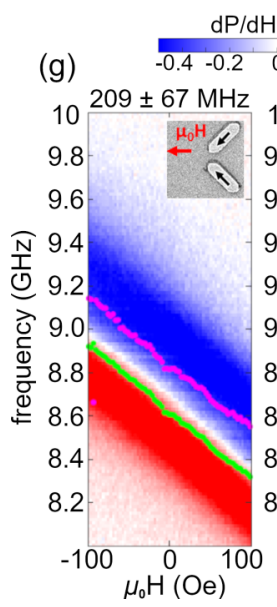

\begin{tabular}{llll}
\hline 2 & 0 & 0.2 & 0.4
\end{tabular}

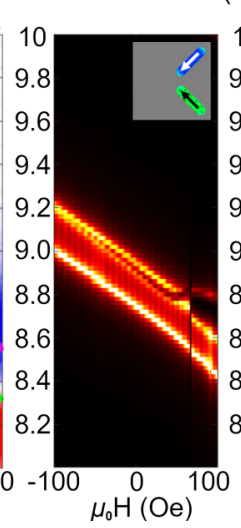

(h)

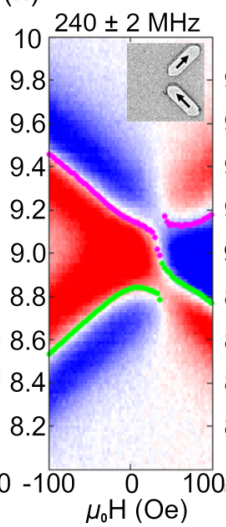

Power (a.u.)

$\begin{array}{lllll}0.2 & 0.4 & 0.6 & 0.8 & 1\end{array}$

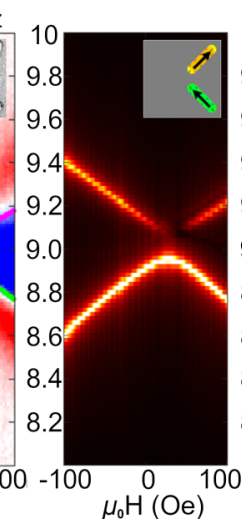

(i)

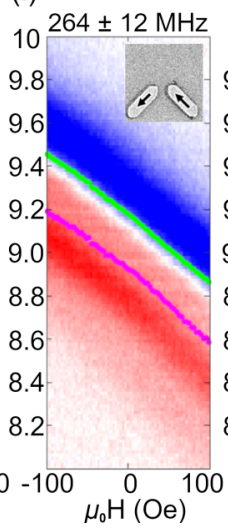

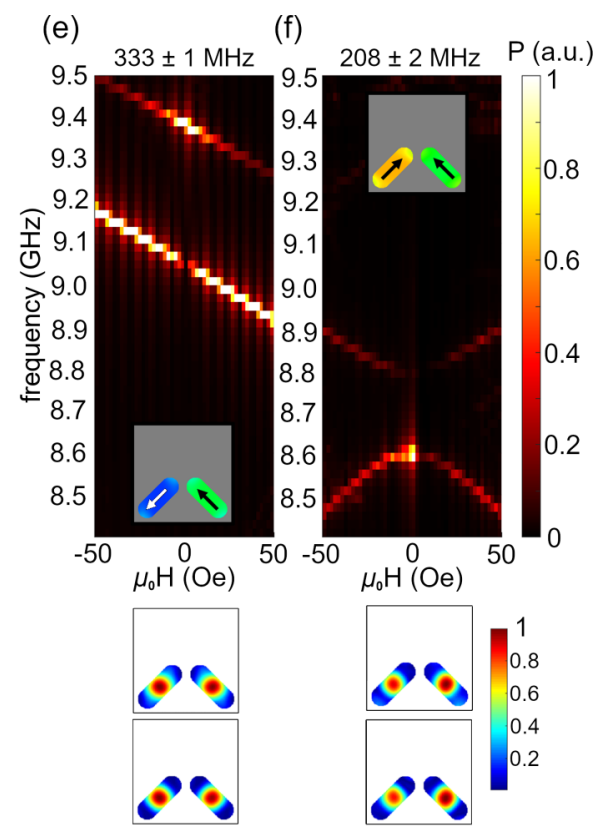

FIG. 2. (a)-(f) Simulated spectra for diagonal [(a), (b)] and chevron [(c)-(f)] two-nanoisland configurations. Microstates are shown inset for each plot, corresponding power and phase maps shown below. Applied field direction is along $x$ axis and swept from positive to negative as indicated by red arrow in (a). (g)-(j) Experimental (blue/white/red) and corresponding simulation (black/red/white) for each microstate/field configuration. Two peaks are not immediately resolvable in (g), (i) but broader tails on high-frequency (g) and low-frequency sides (i) are evidence of a second lower-power peak, consistent with simulations (c) and (e). Lorentzian fits represented by green and pink dots (g)-(j).

microstate in Fig. 1(g) has modes with the same field-gradient sign but should still exhibit a gap-but since $\delta$ is typically on the order of hundreds of $\mathrm{MHz}$, it is likely obscured by the experimental linewidth.

\section{B. Mode hybridization in two-nanoisland arrays}

Three distinct two-nanoisland systems are shown in Fig. 2 with nanoisland dimensions $220 \times 80 \times 20 \mathrm{~nm}$ and lattice parameter $\Lambda=300 \mathrm{~nm}$. Spatial Fourier transforms are applied to a magnetization time series to generate spin-wave spectra, power, and phase maps. The diagonal two-nanoisland system [Figs. 2(a) and 2(b)] demonstrates clear distinction between the $\uparrow \uparrow$ [Figs. 2(a)] and $\uparrow \downarrow$ 2(b) spectra. Figure 2(a) exhibits a single mode, increasing in frequency as the field is swept positive to negative along the $x$ axis. The corresponding power plot underneath shows equal power in both nanoislands and phase plots show both in-plane (top) and out-of-plane (bottom) magnetization precessing in-phase. Figure 2(b) shows the $\uparrow \downarrow$ case exhibiting acoustic and optical modes with an avoided crossing of $\delta=126 \pm 2 \mathrm{MHz}$ at zero field, relatively small due to the center to center distance being $\Lambda$ compared with chevron arrays with $\Lambda / \sqrt{2}$. Power maps appear similar, but phase maps reveal expected optical and acoustic-mode phase relationships. The acoustic mode has in-plane components of magnetization moving in phase and the optical mode has in-plane components moving out of phase.

Next we examine simulated spin-wave spectra of four possible microstates [Figs. 2(c)-2(f)] in a chevron' geometry. In Fig. 2(c), the $x$ component of the magnetization $\left(m_{x}\right)$ is collinear with the swept magnetic field direction and $\delta$ is constant for all fields. Along the $y$-component of magnetization $\left(m_{y}\right)$, one nanoisland points up $\left(+m_{y}\right)$ and the other down $\left(-m_{y}\right)$, satisfying optical and acoustic-mode generation 

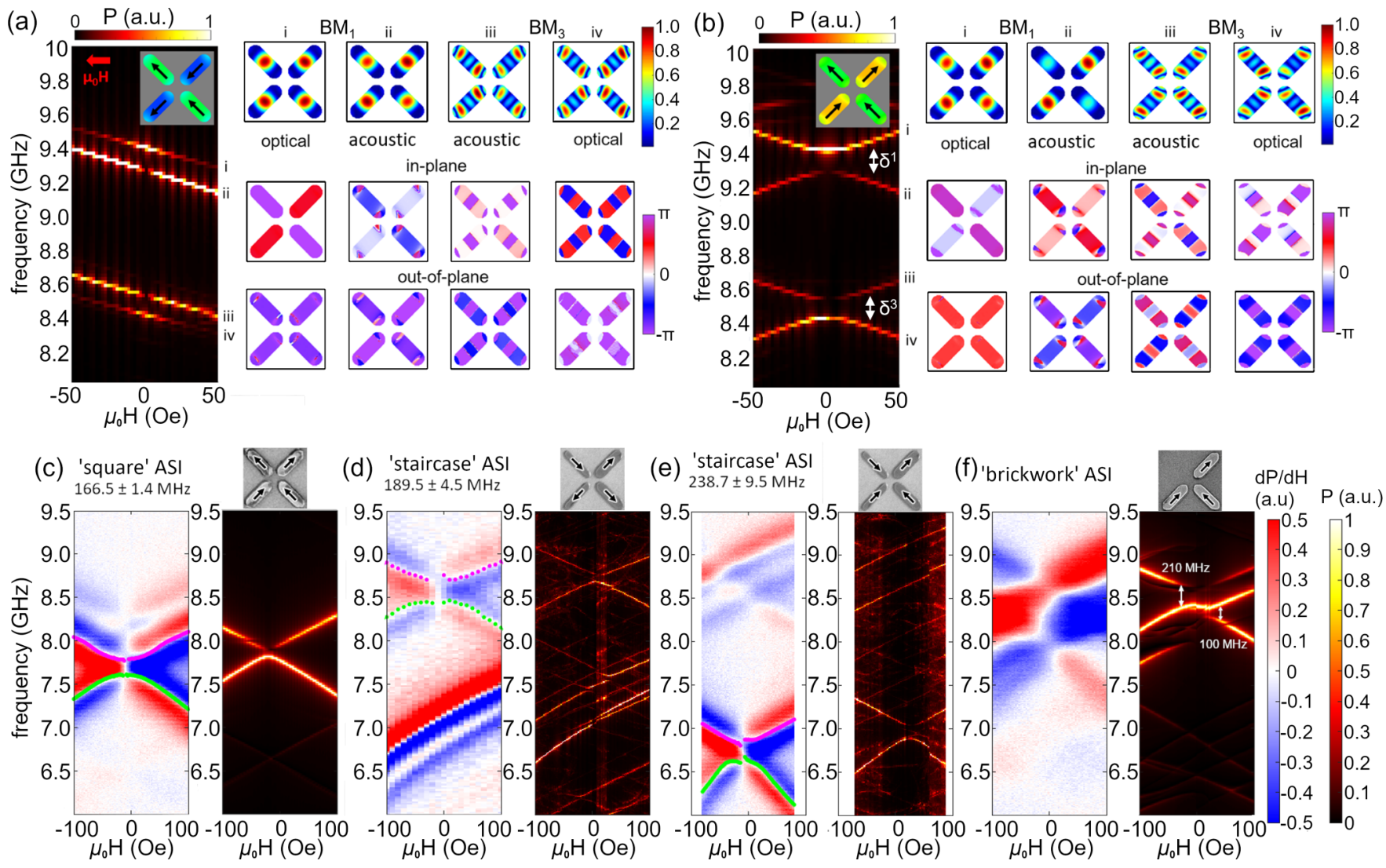

FIG. 3. (a) Spectra for the type-2 microstate shown inset, saturated along the swept field direction (indicated by red arrow). Modes i-iv are indicated on the right side of the spectra. Modes $\mathrm{i}$ and ii are similar to those in Fig. 1 with power concentrated in nanoisland center. Modes $\mathrm{BM}_{3}^{\text {opt }}$ and $\mathrm{BM}_{3}^{\text {aco }}$ have shorter wavelengths and backward-volume magnetostatic spin wave character (BVMSW). (b) Spectra for type-2 microstate saturated perpendicular to the swept-field direction. (c)-(f) Experimental and simulation results for (c) square ASI (d), (e) staircase ASI, and (f) brickwork ASI. Black arrows illustrate microstates.

conditions. The two modes exhibit $\delta=194 \pm 1 \mathrm{MHz}$, higher than Fig. 2(b) since the interisland distance is $\Lambda / \sqrt{2}$. A large $\delta$ of $335 \pm 1 \mathrm{MHz}$ is observed in Fig. 2(d), showing strong interisland mode coupling.

$\delta$ depends not only on the microstate but also the local field which is a function of magnetization alignment favorability. Figures 2(d) and 2(e) are favorably aligned, showing higher overall frequency and $\delta$ compared to Figs. 2(c) and 2(f) which are unfavorably configured. Since the frequency increases with effective field, $\mathbf{H}_{\mathrm{eff}}$, which includes a dipolar-field term $\mathbf{H}_{\text {dip }}$, the cancellation of the dipolar fields when two moments are both pointing into the vertex lowers the resonant frequency. In Figs. 2(c) and 2(e), the upper mode has higher or lower power, respectively, allowing experimental detection via asymmetry in the spin-wave signature.

Figures 2(g)-2(j) are experimental (red/blue) and simulated (black/red/white) results for the fabricated chevron sample with dimensions $540 \times 140 \times 25 \times \mathrm{nm}$ and $\Lambda=800$ $n$ m. The experimental differential FMR heat maps $\left(\frac{\partial P}{\partial H}\right)$ are consistent with simulations. Larger $\delta$ is observed for microstates with favorable alignment in Figs. 2(h) and 2(i) with $\delta=240 \pm 2 \mathrm{MHz}$ and $264 \pm 12 \mathrm{MHz}$, respectively. Unfavorable microstates in Figs. 2(g) and 2(j) have a smaller $\delta$ of 209 $\pm 67 \mathrm{MHz}$ and $229 \pm 2 \mathrm{MHz}$, respectively, demonstrating tunability via microstates. The relative optical and acousticmode power depends on the configuration as discussed above.
The asymmetry of red and blue shading and Lorentzian fitting (green and pink dots) in Figs. 2(g) and 2(i) reveals two modes.

For conventional symmetric ASI in type-2 states, mode shifting due to local field distributions is insignificant. For symmetry-broken type-3 ASI states, brickwork, or widthmodified ASIs, the vertex-driven local fields can be leveraged to tune $\delta$ via field or microstate control.

\section{Mode hybridization in ASIs}

Figure 3 shows simulated spectra for square ASI microstates saturated with the measurement field parallel Fig. 3(a) and perpendicular Fig. 3(b) to the preparation field. In simulations, coupling for the higher order BM is observed and we define the two gaps as $\delta^{1}=f\left(\mathrm{BM}_{1}^{\mathrm{opt}}\right)-f\left(\mathrm{BM}_{1}^{\mathrm{aco}}\right)=$ $146 \pm 2 \mathrm{MHz}$ and $\delta^{3}=f\left(\mathrm{BM}_{3}^{\mathrm{opt}}\right)-f\left(\mathrm{BM}_{3}^{\mathrm{aco}}\right)=127 \pm$ $2 \mathrm{MHz} . \mathrm{BM}_{3}$ are present in experiment but too faint to make quantitative assessment (see Supplemental Material [44]).

Modes i and ii in Figs. 3(a) and 3(b) follow similar phase relationships as the two-island case. Remembering that the $\mathrm{BM}_{1}^{\mathrm{opt}}$ has in-plane magnetization of coupled nanoislands moving out of phase with each other, for $\mathrm{BM}_{1}^{\text {aco }}$ the phase of the precession of coupled nanoislands move in-phase. $\mathrm{BM}_{3}$ are best described by backward volume magnetostatic spin waves (BVMSWs) [45], where the wave vector and 
(a)

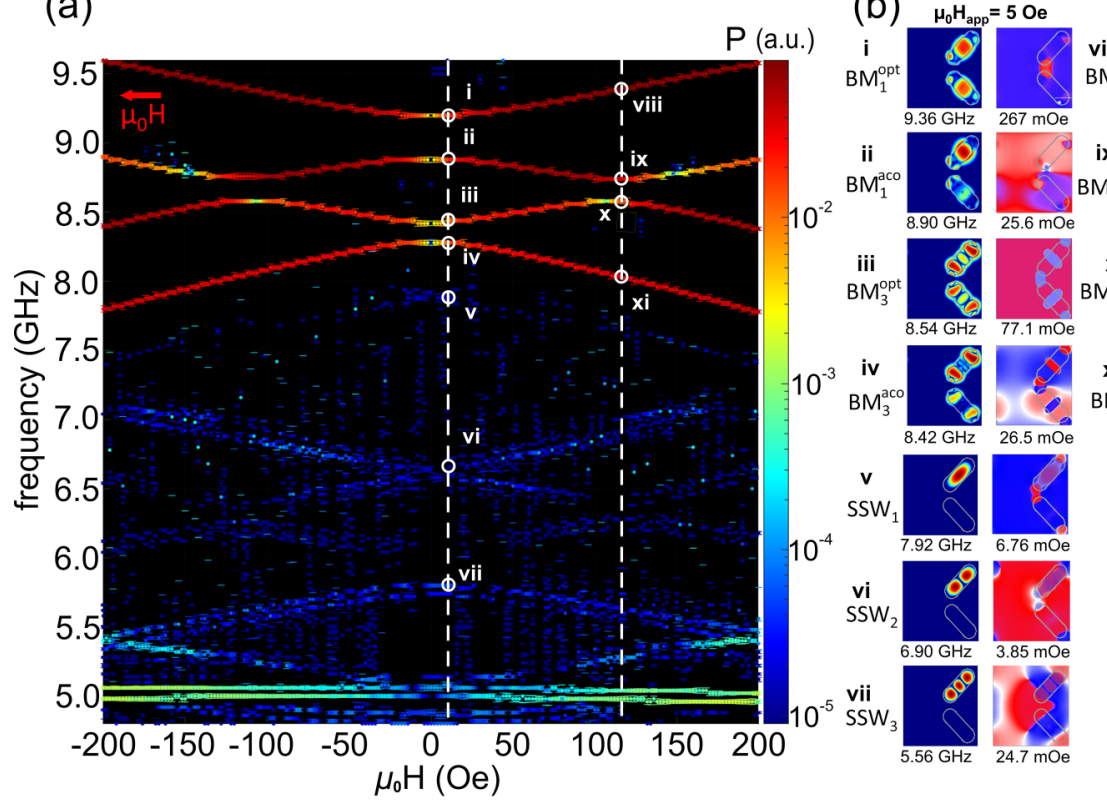

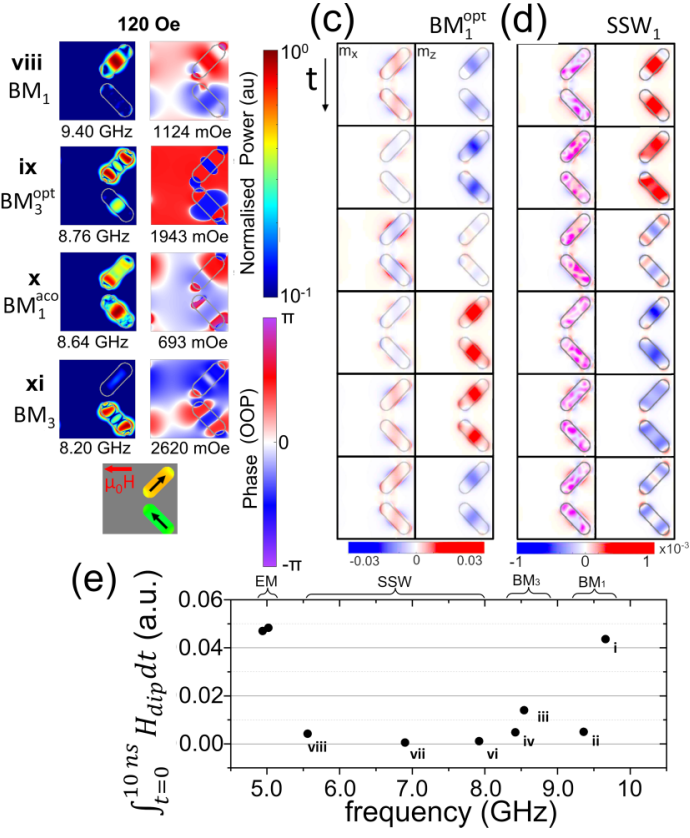

FIG. 4. (a) Peak extraction of $\pm 20 \mathrm{mT}$ high frequency-resolution sweep for same chevron state in Fig. 2(d). Avoided crossings are observed at higher fields between modes $\mathrm{BM}_{1}$ (ix) and $\mathrm{BM}_{3}$ (x). (b) Power and out-of-plane phase maps for each of the labeled modes at 5 Oe and 120 Oe also indicated by white dotted lines in (a). Analysis is applied to demagnetizing field to visualize dynamic stray field. Stray-field value is displayed under phase maps. Time-domain simulations for (c) $\mathrm{BM}_{1}^{\mathrm{opt}}$ and (d) $\mathrm{SSW}_{1}$ mode where one precession cycle is shown. Precession for $\mathrm{SSW}_{1}$ is purely out of plane. $\mathrm{BM}_{1}^{\mathrm{opt}}$ shows more coherent in-plane mode structure and is a combination of EM and SSW. (e) Stray-field outside nanoisland is integrated over time and plotted for each mode.

magnetization are both defined parallel to the nanoisland long axis. $\mathrm{BM}_{3}^{\text {opt }}$ and $\mathrm{BM}_{3}^{\text {aco }}$ have lower frequencies than the $\mathrm{BM}_{1}$, as expected for BVMSWs [49]. In Fig. 3(b), the lower power of $\mathrm{BM}_{1}^{\mathrm{aco}}$ is due to the homogeneous excitation field inefficiently driving a mode where out-of-phase precession is the resonant condition.

Experimentally measured and simulated FMR spectra for four ASI cases exhibiting collective spin-wave signatures are compared: square ASIs in a perpendicular type- 2 state, staircase ASIs [15] in type-3 states; wide-nanoisland and thin-nanoisland majority magnetization and perpendicularly saturated brickwork ASIs [Fig. 3(f)].

Figure 3(c) shows spectra for symmetric square ASIs with dimensions $460 \times 150 \times 25 \mathrm{~nm}$ and $\Lambda=600 \mathrm{~nm} . \delta=$ $166.5 \pm 1.4 \mathrm{MHz}$, demonstrating avoided crossings, are experimentally resolved in ASIs without differential fabrication.

Figures 3(d) and 3(e) show spectra for a staircase ASI with dimensions $600 \times 200$ (wide) $/ 130$ (thin) $\times 20 \mathrm{~nm}$ and $\Lambda=800 \mathrm{~nm}$. Figure $3(\mathrm{~d})$ shows $\delta^{1}=189.5 \pm 4.45 \mathrm{MHz}$, and Fig. 3(e), $\delta^{1}=238.7 \pm 9.5 \mathrm{MHz}$. Preparing the widenanoisland majority type- 3 state locates the avoided-crossing in the high-frequency, hybridized thin-nanoisland modes. The thin-nanoisland majority type- 3 exhibits avoided crossing in the low-frequency, wide-nanoisland hybridized modes. This demonstrates tunability of $\delta^{1}$ via microstate switching for RMC applications.

Figure 3(f) shows brickwork ASI with dimensions $570 \times$ $170 \times 25 \mathrm{~nm}$ and $\Lambda=800 \mathrm{~nm}$ achieved via single nanoisland removal from the unit cell. The simulated avoided-crossing gaps were $\delta^{1}=240 \pm 25 \mathrm{MHz}$ in the negative field region (right coupled nanoislands) and $\delta^{1}=100 \pm 25 \mathrm{MHz}$ in the positive field region (bottom coupled nanoislands). Unfortunately, accurate experimental peaks were not extracted due to larger linewidths, however, the presence of avoided crossings is plausible based on the similarity to the simulated spectra. Observing two different $\delta^{1}$ is consistent with the chevron findings and allows tunability via field without microstate change. Lorentzian fits to experimental data are available in Supplemental Material [44].

\section{Mode profiles}

Spatial Fourier transforms are applied to the demagnetization field to show dynamic stray-field profiles. Peak extractions are plotted in Fig. 4(a) to better highlight weak peaks. $\mathrm{BM}_{1}$ and $\mathrm{BM}_{3}$ also exhibit an avoided crossing at 120 Oe as indicated by ix and $\mathrm{x}$. At this point, $\mathrm{BM}_{3}$ is optical and $\mathrm{BM}_{1}$ is acoustic. $\mathrm{BMs}$ revert to single nanoisland-mode behavior at high fields. Figure 4(b) shows the corresponding power and phase of each mode labeled in Fig. 4(a). $\mathrm{SSW}_{1-3}$ have a significantly lower dynamic dipole field confined to the nanoisland volume, agreeing with the theoretical assumption that no coupling for $\mathrm{SSW}_{1-3}$ is expected. Values shown underneath each phase plot in Fig. 4(b) are dipole-field calculated outside the nanoislands. Modes v and vi (noncoupled SSWs) show significantly smaller values than i-iv (coupled BM). $\mathrm{BM}_{1}$ and $\mathrm{BM}_{3}$ power is clearly distributed across multiple nanoislands, contrasted with SSWs localized to single nanoislands.

The SSW picture applies well to low-frequency modes that are not experimentally detected. Experimentally detected BMs are best described by the combination of EMs and SSWs 
indicating geometry, particularly the nanoisland ends, plays a significant role. Additionally, there is a clear manifestation of stray field emanating from long nanoisland edges.

We excite modes sinusoidally to examine time-domain dynamics. Snapshots of demagnetizing fields over a full precession cycle are shown in Figs. 4(c) and 4(d) for the $\mathrm{BM}_{1}^{\mathrm{opt}}$ and $\mathrm{SSW}_{1}$ modes, respectively. All modes are available in Supplemental Material video $1 . \mathrm{BM}_{1}^{\mathrm{opt}}$ shows coherent dynamics for the in-plane components whereas mode $\mathrm{SSW}_{1}$ shows precession in $m_{z}$ only. Coherent in-plane precession for $\mathrm{BM}_{1}^{\mathrm{opt}}$ fosters internanoisland coupling. The video shows the EM and SSW components of the BM have a transverse and longitudinal character, respectively, seemingly arising due to the curved geometry at nanoisland ends.

Figure 4(e) shows the stray field outside magnetic volume integrated over $10 \mathrm{~ns} . \mathrm{BM}_{1}^{\mathrm{opt}}$ has a comparable stray field to the two EMs, further indicating edges are vital to BM coupling. Increasing the node number for SSWs allows more stray field to escape for potential coupling to occur; however, even in the simulations SSWs are far too weak to be experimentally detected.

\section{E. Tailoring coupling via geometry}

Tunability of frequency gaps between $\mathrm{BM}_{1}\left(\delta^{1}\right)$ and $\mathrm{BM}_{3}$ $\left(\delta^{3}\right)$ modes via parameter exploration is investigated using micromagnetic simulation. The control case is a square ASI with $220 \times 80 \times 20 \mathrm{~nm}, \Lambda=300 \mathrm{~nm}$, and $M_{\text {sat }}=750 \mathrm{kA} / \mathrm{m}$.

Figure 5(a) shows increasing nanoisland length 160$240 \mathrm{~nm}$ increases coupling strength $0-200 \mathrm{MHz}$. This is a function of nanoisland ends being in closer proximity and larger magnetic volume. Wave vectors may also play a role in determining how much stray field escapes the nanoisland.

Figure 5(b) shows $\delta^{1}$ as a function of nanoisland width which remains relatively constant. $\delta^{1}$ disappears at $146 \mathrm{~nm}$ due to distortions arising from an ill-defined magnetization vector (see Supplemental Material [44]). Wider nanoislands prevent the typical BM structure observed elsewhere, behaving more like a macrospin with decreased stray field. Interestingly, $\delta^{3}$ depends strongly on width. The multinodal structure of the higher-order mode seems more robust and maintains its stray field. The antinode edge proximity increases coupling significantly. The dotted line indicates the point at which neighboring nanoislands become connected and different mode behavior is observed.

Figure 5(c) shows how $\delta$ varies with thickness. A minimum thickness of $\sim 14 \mathrm{~nm}$ is required for measurable coupling, above which $\delta^{1}$ and $\delta^{3}$ increase up to constant value at around $20 \%$ of nanoisland width, showing additional modes at large thicknesses. Intersection with a high-order EM interrupts $\delta^{1}$ for 25-27 nm and continues to increase thereafter up to $35 \mathrm{~nm}$ where additional modes interfere with the $\mathrm{BM}_{1}$. There is an avoided-crossing at $15 \mathrm{~nm}$, indicated by the dotted line. Figure 4 previously demonstrated that a frequency gap occurs between $\mathrm{BM}_{3}$ and $\mathrm{BM}_{1}$. For $14 \mathrm{~nm}$ and below, the $\mathrm{BM}_{3}$ have a higher frequency than $\mathrm{BM}_{1}$. Each of these avoided crossings is explained by mode hybridization confirmed by significant stray field (see Supplemental Material [44]). Below $14 \mathrm{~nm}$, $\mathrm{BM}_{3}$ resemble magnetostatic surface spin waves, where they exhibit a higher frequency than $\mathrm{BM}_{1}$ [49].
Figure $5(\mathrm{~d})$ shows $\delta$ varying with lateral scaling achieved by an in-plane cell-size increase. $\delta^{1}$ decreases linearly with lateral scaling as volume increases as the square and dipoledipole strength decreases as the cube resulting in a linear decrease overall. However, $\delta^{1}$ is significantly decreased when intersecting with other modes at $0.8,1.4$, and 2.0. $\delta^{3}$ seems much less sensitive to the scaling parameter than $\delta^{1}$ above 1 . As can be seen in Fig. 4(b), the maximal precession location of $\mathrm{BM}_{3}$ is closer to the nanoisland ends. The magnetic volume increases more than the coupling distance, therefore exhibiting a much more shallow drop compared to $\delta^{1}$.

Figure 5(e) shows $\delta^{1}$ and $\delta^{3}$ both initially increase rapidly with $M_{\text {sat }}$ with diminishing returns. These findings imply that materials with higher saturation magnetization like $\mathrm{CoFeB}$ could express significantly larger spin-wave band gaps.

Finally, Fig. 5(f) shows how $\delta^{1}$ and $\delta^{3}$ decrease rapidly with increasing lattice parameter. This is strong evidence that the coupling is dipole mediated as it decreases with the cube and modes become decoupled when the interisland distance is greater than about twice the nanoisland length.

\section{CONCLUSION}

We investigated and explained the origin of avoided crossings as hybridization of single nanoisland modes into acoustic and optical modes which can be distinguished by precession phase relationships. Hybridization between $\mathrm{BM}_{1}$ and $\mathrm{BM}_{3}$ also occurs at higher fields.

Avoided crossings in artificial spin systems previously remained elusive due to typical experimental approaches employing preparation and measurement fields along the same axis. Rotating the applied field perpendicular to the preparation field or preparing broken-symmetry microstates (i.e., type-3) generates a clear gap, $\delta$. Conversely, parallel preparation and detection field direction exhibit, $\delta$, often hidden by relatively large experimental linewidths. Theoretically, it was assumed that insufficient stray field between nanoislands prevents effective coupling. We showed using simulation that this holds for SSW modes [Figs. 4(b)v-4(b)vii]. However, experimentally detected BMs are a combination of SSWs and EMs, exhibiting significant stray field shown in Figs. 4(b)i-4(b)iv and avoided crossings are confirmed via FMR. Nanoisland ends play a significant role in determining dipolar-coupling strength even between BMs.

The work presented here progresses the understanding of dynamic dipole-dipole coupling in nanomagnetic arraysboth its fundamental origin and how to harness and design it into systems. Much proposed magnonic computing is based on spin-wave interference effects in continuous magnetic media. Here we showed discrete nanopatterned islands can strongly interfere through collective-hybridization-allowing interference effects with the reconfigurability and flexibility benefits of 2D-RMC. The nascent magnonic computing field requires heuristics of how to optimize systems for maximum interference, coupling, and nonlinearity [24]. The design rules presented here provide means for this in nanopatterned 2D ASI-based RMC. There are multiple ways of tuning $\delta$ in the same structure via field application or microstate selection, proving ASI and related structures to be promising candidates. We hope that unveiling the ability to generate 

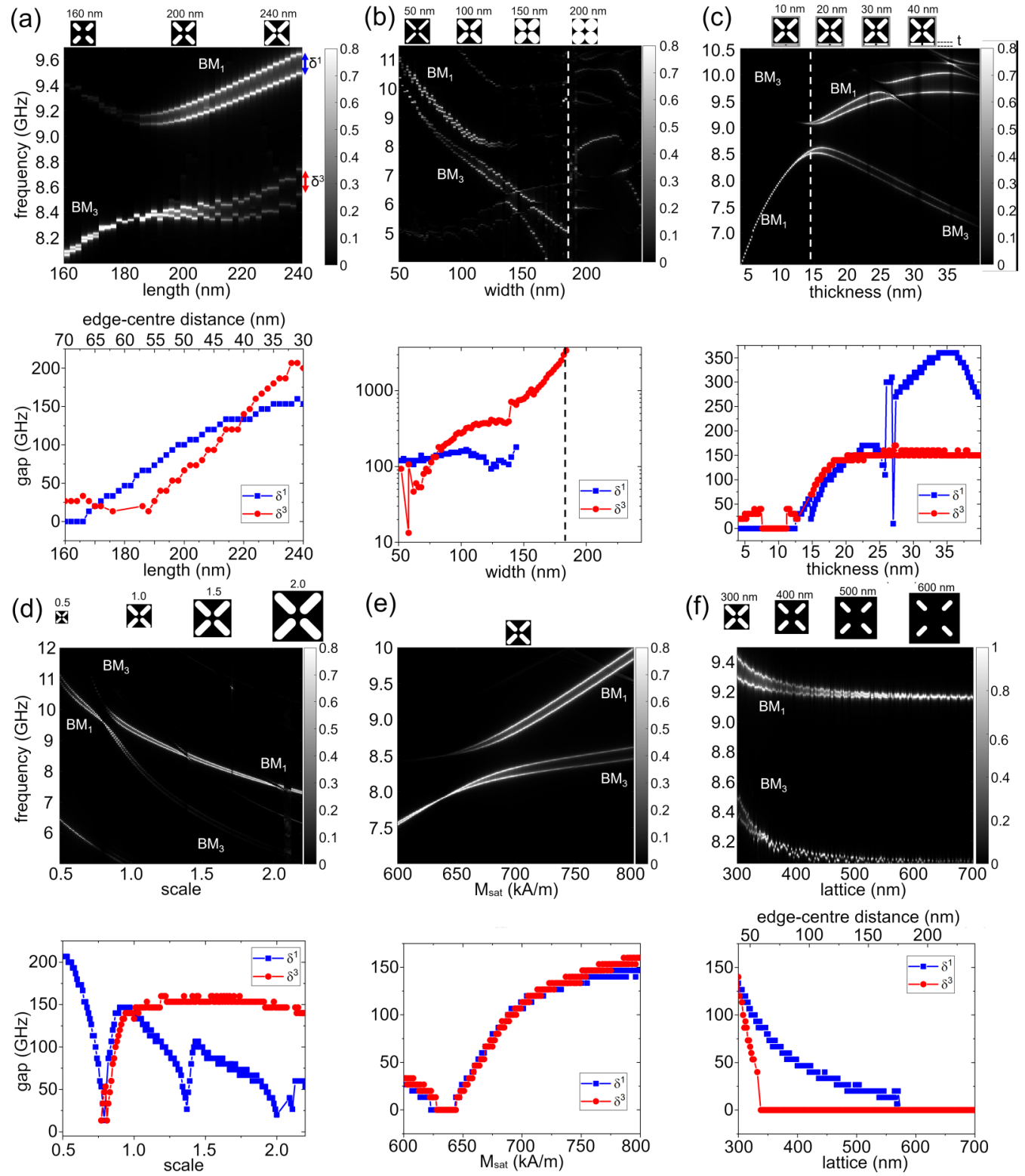

FIG. 5. Upper panels show the spectra and lower panels show the extracted $\delta$ for the $\delta^{0}\left(f_{i}-f_{i i}\right)$ and $\delta^{1}\left(f_{i i i}-f_{i v}\right)$ modes as a function of (a) nanoisland length, (b) width, (c) thickness, (d), lateral scaling, (e) saturation magnetization, and (f) lattice parameter. Insets show geometry being changed and are all to scale with (e) showing geometry common to all panels. The dotted line in (c) indicates the point where the $\mathrm{BM}_{1}^{\mathrm{opt}}$ and $\mathrm{BM}_{1}^{\mathrm{aco}}$ modes switch positions in frequency with the higher order $\mathrm{BM}_{3}^{\mathrm{aco}}$ and $\mathrm{BM}_{3}^{\mathrm{opt}}$ modes. This may be treated as another avoided crossing.

and observe these avoided crossings will encourage future studies into dipolar magnon-magnon coupling and collective mode-hybridization in artificial spin systems and other architectures.

Code and data sets generated during and/or analyzed during the current study are available from the corresponding author on reasonable request.

\section{ACKNOWLEDGMENTS}

T.D. was supported by the International Research Fellow of Japan Society for the Promotion of Science (Postdoctoral Fellowships for Research in Japan). W.R.B. acknowledges support from the Leverhulme Trust (No. RPG-2017-257).
A.V. was supported by the EPSRC Centre for Doctoral Training in Advanced Characterisation of Materials (Grant No. $\mathrm{EP} / \mathrm{L} 015277 / 1)$. Simulations were performed on the Imperial College London Research Computing Service [50]. The authors would like to thank Professor Lesley F. Cohen of Imperial College London for enlightening discussion and comments, and David Mack for excellent laboratory management.

The authors declare no competing interests.

T.D. and J.C.G. conceived the paper. J.C.G., K.D.S., and A.V. fabricated the samples. A.V. and J.C.G. performed CAD design of the structures. J.C.G., A.V., and K.D.S. performed FMR measurements. J.C.G. and A.V. performed analysis of FMR measurements. T.D. performed 
simulations and analysis. H.K. and D.M.A. supported analysis of spin-wave spectra and interpretation. T.D. drafted the paper, with contributions from all authors in editing and revision stages.
[1] R. F. Wang, C. Nisoli, R. S. Freitas, J. Li, W. McConville, B. J. Cooley, M. S. Lund, N. Samarth, C. Leighton, V. H. Crespi et al., Artificial "spin ice" in a geometrically frustrated lattice of nanoscale ferromagnetic islands, Nature (London) 439, 303 (2006).

[2] C. Nisoli, R. Moessner, and P. Schiffer, Colloquium: Artificial spin ice: Designing and imaging magnetic frustration, RMP 85, 1473 (2013).

[3] S. H. Skjærvø, C. H. Marrows, R. L. Stamps, and L. J. Heyderman, Advances in artificial spin ice, Nat. Rev. Phys. 2, 13 (2020).

[4] J. C. Gartside, D. M. Arroo, D. M. Burn, V. L. Bemmer, A. Moskalenko, L. F. Cohen, and W. R. Branford, Realization of ground state in artificial kagome spin ice via topological defectdriven magnetic writing, Nat. Nanotechnol. 13, 53 (2018).

[5] M. T. Kaffash, S. Lendinez, and M. B. Jungfleisch, Nanomagnonics with artificial spin ice, Phys. Lett. A 402, 127364 (2021).

[6] A. Barman, S. Mondal, S. Sahoo, and A. De, Magnetization dynamics of nanoscale magnetic materials: A perspective, J. Appl. Phys. 128, 170901 (2020).

[7] S. Lendinez and M. Jungfleisch, Magnetization dynamics in artificial spin ice, J. Condens.: Matter Phys. 32, 013001 (2019).

[8] S. Gliga, E. Iacocca, and O. G. Heinonen, Dynamics of reconfigurable artificial spin ice: Toward magnonic functional materials, APL Mater. 8, 040911 (2020).

[9] A. Talapatra, N. Singh, and A. O. Adeyeye, Magnetic Tunability of Permalloy Artificial Spin Ice Structures, Phys. Rev. Appl. 13, 014034 (2020).

[10] J.-C. Gartside, S. G. Jung, S. Y. Yoo, D. M. Arroo, A. Vanstone, T. Dion, K. D. Stenning, and W. R. Branford, Current-controlled nanomagnetic writing for reconfigurable magnonic crystals, Commun. Phys. 3, 1 (2020).

[11] J. Grollier, D. Querlioz, K. Camsari, K. Everschor-Sitte, S. Fukami, and M. Stiles, Neuromorphic spintronics, Nat. Electron. 3, 360 (2020).

[12] K. Hon, Y. Kuwabiraki, M. Goto, R. Nakatani, Y. Suzuki, and H. Nomura, Numerical simulation of artificial spin ice for reservoir computing, Appl. Phys. Express 14, 033001 (2021).

[13] H. Nomura, T. Furuta, K. Tsujimoto, Y. Kuwabiraki, F. Peper, E. Tamura, S. Miwa, M. Goto, R. Nakatani, and Y. Suzuki, Reservoir computing with dipole-coupled nanomagnets, Jpn. J. Appl. Phys. 58, 070901 (2019).

[14] J. H. Jensen and G. Tufte, Reservoir computing in artificial spin ice, in Artificial Life Conference Proceedings (MIT Press, MIT Press Direct, Cambridge, MA 02142, 2020), pp. 376-383.

[15] J. C. Gartside, K. D. Stenning, A. Vanstone, T. Dion, H. H. Holder, D. M. Arroo, F. Caravelli, H. Kurebayashi, and W. R. Branford, Reconfigurable training and reservoir computing in an artificial spin-vortex ice via spin-wave fingerprinting, arXiv:2107.08941v3.

[16] T. Dion, D. M. Arroo, K. Yamanoi, T. Kimura, J. C. Gartside, L. F. Cohen, H. Kurebayashi, and W. R. Branford, Tunable magnetization dynamics in artificial spin ice via shape anisotropy modification, Phys. Rev. B 100, 054433 (2019).
[17] J. C. Gartside, A. Vanstone, T. Dion, K. D. Stenning, D. M. Arroo, H. Kurebayashi, and W. R. Branford, Reconfigurable magnonic mode-hybridization and spectral control in a bicomponent artificial spin ice, Nat. Commun. 12, 2488 (2021).

[18] A. Vanstone, J. C. Gartside, K. D. Stenning, T. Dion, D. M. Arroo, and W. R. Branford, Spectral-fingerprinting: Microstate readout via remanence ferromagnetic resonance in artificial spin systems, arXiv:2106.04406.

[19] S. Lendinez, M. T. Kaffash, and M. B. Jungfleisch, Emergent spin dynamics enabled by lattice interactions in a bicomponent artificial spin ice, Nano Lett. 21, 1921 (2021).

[20] D. Grundler, Reconfigurable magnonics heats up, Nat. Phys. 11, 438 (2015).

[21] J. Topp, D. Heitmann, M. P. Kostylev, and D. Grundler, Making a Reconfigurable Artificial Crystal by Ordering Bistable Magnetic Nanowires, Phys. Rev. Lett. 104, 207205 (2010).

[22] M. Krawczyk and D. Grundler, Review and prospects of magnonic crystals and devices with reprogrammable band structure, J. Phys.: Condens. Matter 26, 123202 (2014).

[23] A. Haldar, D. Kumar, and A. O. Adeyeye, A reconfigurable waveguide for energy-efficient transmission and local manipulation of information in a nanomagnetic device, Nat. Nanotechnol. 11, 437 (2016).

[24] A. Chumak, P. Kabos, M. Wu, C. Abert, C. Adelmann, A. Adeyeye, J. Åkerman, F. Aliev, A. Anane, A. Awad et al., Roadmap on spin-wave computing concepts, arXiv:2111.00365

[25] J. Topp, G. Duerr, K. Thurner, and D. Grundler, Reprogrammable magnonic crystals formed by interacting ferromagnetic nanowires, Pure Appl. Chem. 83, 1989 (2011).

[26] Z. K. Wang, V. L. Zhang, H. S. Lim, S. C. Ng, M. H. Kuok, S. Jain, and A. O. Adeyeye, Observation of frequency band gaps in a one-dimensional nanostructured magnonic crystal, Appl. Phys. Lett. 94, 83112 (2009).

[27] H. Al-Wahsh, A. Akjouj, B. Djafari-Rouhani, and L. Dobrzynski, Magnonic circuits and crystals, Surf. Sci. Rep. 66 , 29 (2011).

[28] F. S. Ma, H. S. Lim, V. L. Zhang, S. C. Ng, and M. H. Kuok, Magnonic band structure investigation of one-dimensional bicomponent magnonic crystal waveguides, Nanoscale Res. Lett. 7, 498 (2012).

[29] G. Gubbiotti, S. Tacchi, G. Carlotti, P. Vavassori, N. Singh, S. Goolaup, A. O. Adeyeye, A. Stashkevich, and M. Kostylev, Magnetostatic interaction in arrays of nanometric permalloy wires: A magneto-optic kerr effect and a brillouin light scattering study, Phys. Rev. B 72, 224413 (2005).

[30] G. Gubbiotti, S. Tacchi, G. Carlotti, N. Singh, S. Goolaup,A. O. Adeyeye, and M. Kostylev, Collective spin modes in monodimensional magnonic crystals consisting of dipolarly coupled nanowires, Appl. Phys. Lett. 90, 092503 (2007).

[31] D. M. Arroo, J. C. Gartside, and W. R. Branford, Sculpting the spin-wave response of artificial spin ice via microstate selection, Phys. Rev. B 100, 214425 (2019).

[32] V. Kruglyak, S. Demokritov, and D. Grundler, Magnonics, J. Phys. D 43, 264001 (2010). 
[33] B. Lenk, H. Ulrichs, F. Garbs, and M. Münzenberg, The building blocks of magnonics, Phys. Rep. 507, 107 (2011).

[34] A. V. Chumak, V. I. Vasyuchka, A. A. Serga, and B. Hillebrands, Magnon spintronics, Nat. Phys. 11, 453 (2015).

[35] Y. Shiota, T. Taniguchi, M. Ishibashi, T. Moriyama, and T. Ono, Tunable Magnon-Magnon Coupling Mediated by Dynamic Dipolar Interaction in Synthetic Antiferromagnets, Phys. Rev. Lett. 125, 017203 (2020).

[36] A. Sud, C. W. Zollitsch, A. Kamimaki, T. Dion, S. Khan, S. Iihama, S. Mizukami, and H. Kurebayashi, Tunable magnonmagnon coupling in synthetic antiferromagnets, Phys. Rev. B 102, 100403(R) (2020).

[37] S. Gliga, A. Kákay, R. Hertel, and O. G. Heinonen, Spectral Analysis of Topological Defects in an Artificial Spin-Ice Lattice, Phys. Rev. Lett. 110, 117205 (2013).

[38] Y. Li, G. Gubbiotti, F. Casoli, S. A. Morley, F. J. Gonçalves, M. C. Rosamond, E. H. Linfield, C. H. Marrows, S. McVitie, and R. L. Stamps, Thickness dependence of spin wave excitations in an artificial square spin ice-like geometry, J. Appl. Phys. 121, 103903 (2017).

[39] L. J. Heyderman and R. L. Stamps, Artificial ferroic systems: novel functionality from structure, interactions and dynamics, J. Phys.: Condens. Matter 25, 363201 (2013).

[40] M. P. Kostylev, G. Gubbiotti, J. G. Hu, G. Carlotti, T. Ono, and R. L. Stamps, Dipole-exchange propagating spin-wave modes in metallic ferromagnetic stripes, Phys. Rev. B 76, 054422 (2007).

[41] M. Urbanek, V. Uhlr, P. Babor, E. Kolibalova, T. Hrnir, and J. Spousta, and T. Sikola, Focused ion beam fabrication of spintronic nanostructures: An optimization of the milling process, Nanotechnology 21, 145304 (2010).

[42] E. Iacocca, S. Gliga, and O. Heinonen, Tailoring Spin-Wave Channels in a Reconfigurable Artificial Spin Ice, Phys. Rev. Appl. 13, 044047 (2020).
[43] A. Vansteenkiste, J. Leliaert, M. Dvornik, M. Helsen, F. GarciaSanchez, and B. Van Waeyenberge, The design and verification of MuMax3, AIP Adv. 4, 107133 (2014).

[44] See Supplemental Material at http://link.aps.org/supplemental/ 10.1103/PhysRevResearch.4.013107 for sample fabrication, simulation and experimental methodologies, experimental fits, sample SEM images and additional power maps that complement Fig. 4.

[45] B. Kalinikos and A. Slavin, Theory of dipole-exchange spin wave spectrum for ferromagnetic films with mixed exchange boundary conditions, J. Phys. C: Solid State Phys. 19, 7013 (1986).

[46] L. Liensberger, A. Kamra, H. Maier-Flaig, S. Geprägs, A. Erb, S. T. B. Goennenwein, R. Gross, W. Belzig, H. Huebl, and M. Weiler, Exchange-Enhanced Ultrastrong Magnon-Magnon Coupling in a Compensated Ferrimagnet, Phys. Rev. Lett. 123, 117204 (2019).

[47] Y. Li, G. Gubbiotti, F. Casoli, F. J. Gonçalves, S. A. Morley, M. C. Rosamond, E. H. Linfield, C. H. Marrows, S. McVitie, and R. L. Stamps, Brillouin light scattering study of magnetic-element normal modes in a square artificial spin ice geometry, J. Phys. D: Appl. Phys. 50, 015003 (2016).

[48] M. B. Jungfleisch, W. Zhang, E. Iacocca, J. Sklenar, J. Ding, W. Jiang, S. Zhang, J. E. Pearson, V. Novosad, J. B. Ketterson, O. Heinonen, and A. Hoffmann, Dynamic response of an artificial square spin ice, Phys. Rev. B 93, 100401(R) (2016).

[49] A. V. Chumak, A. A. Serga, B. Hillebrands, and M. P. Kostylev, Scattering of backward spin waves in a onedimensional magnonic crystal, Appl. Phys. Lett. 93, 22508 (2008).

[50] Imperial College rResearch Computing Service, https://doi.org/ $10.14469 / \mathrm{hpc} / 2232$. 